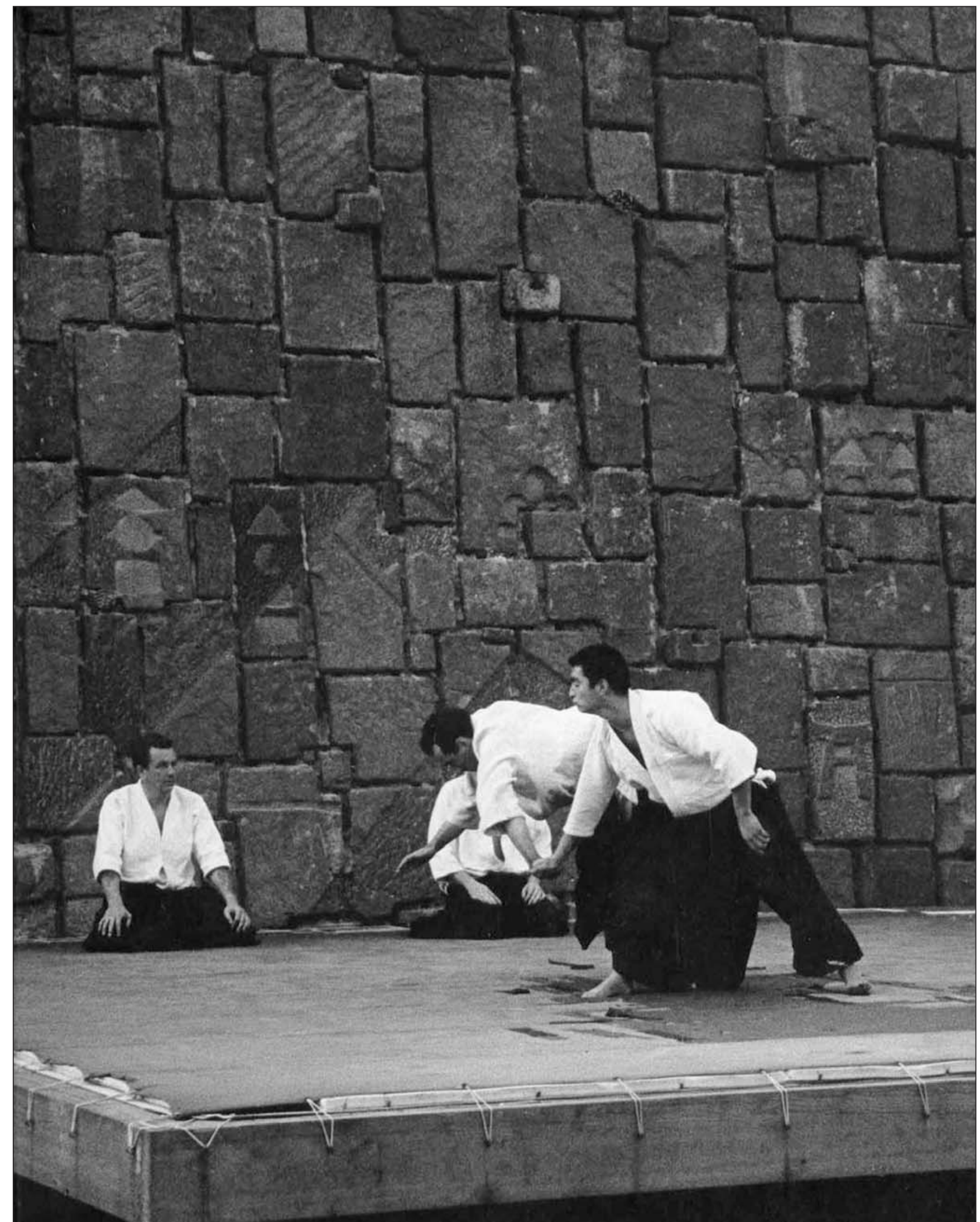

YAMADA YOSHIMITSU EN UNA DEMOSTRACIÓN DE AIKIDO DURANTE la EXPOSICIÓN MUNDial DE NuEVA YoRK DE 1964. 


\section{LA INFLUENCIA DE}
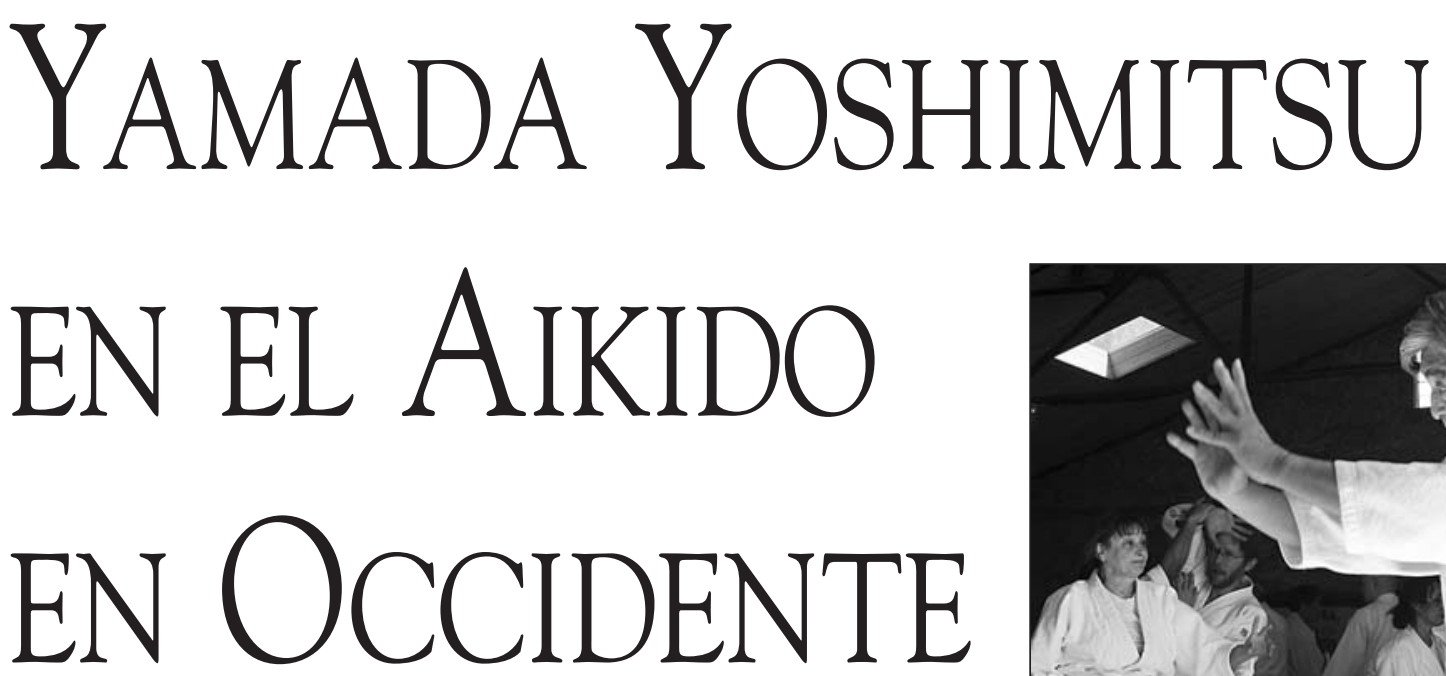

George KenNedy

$\Delta \nabla \mathbf{\Delta}$

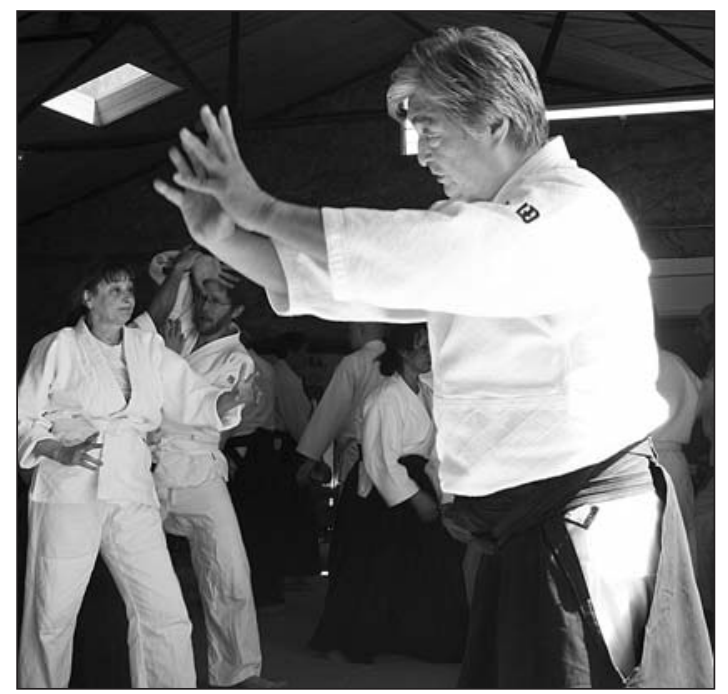

\section{INTRODUCCIÓN}

En la primavera de 1974 asistí a mi primer seminario de aikido. Yo era un joven idealista con poco más de un año de entrenamiento bajo mi cinturón blanco. El seminario tuvo lugar en el dojo de mi localidad en Atlanta (Georgia) bajo la dirección de mi profesor, Rodney Grantham. Los instructores invitados fueron Ueshiba Kisshomaru (hijo del fundador del aikido Ueshiba Morihei) junto con Yamada Yoshimitsu y Kanai Mitsunari, ambos antiguos discípulos (uchi deshi) del fundador. Habría aproximadamente cincuenta personas asistiendo al curso, en representación de toda la región sureste de los Estados Unidos. Sería innecesario decir que todos estábamos amedrentados ante estos maestros Japoneses que sin esfuerzo podían derribar a múltiples agresores a su alrededor mientras irradiaban un aura de serena calma. Supimos que éramos especialmente afortunados por ser asignados a Yamada Yoshimitsu, quién había organizado la primera visita de Ueshiba Kisshomaru a la parte continental de los Estados Unidos.

Yamada había llegado a los Estados Unidos en 1964 para enseñar este arte desconocido en la Exposición Mundial de Nueva York, y reuniendo a un puñado de estudiantes, se quedó para llegar a ser el Instructor Principal del New York Aikikai. Poco tiempo después fundó la Federación Estadounidense de Aikido, que ahora engloba a más de cuatrocientas escuelas de aikido en el norte y en el sur de América. Yamada sigue viajando intensamente, impartiendo más de treinta seminarios por año en países que van desde la punta sur de Sudamérica hasta las extensiones del norte de la antigua Unión Soviética.

Revista de Artes Marciales Asiáticas 9 Volumen 1 Número 1 (48-61) - 2006
YAMADA ENSEÑANDO EN El Aikido Center of Atlanta, MARZO DE 2005.

Todas las fotografías son cortesía de Yamada Yoshimitsu y George Kennedy, excepto las señaladas. 
Demostración DE YAMAdA EN JAPÓN, CERCA DE 1959.

En agosto de 2004, me uní a más de mil personas de todos los continentes que celebraban en el estado de Nueva York los 40 años de Yamada en los Estados Unidos, así como su enorme contribución en la propagación de este arte. Este artículo relata brevemente la historia del impactó que ha tenido esta gran persona en la divulgación del aikido moderno, transformado desde un oscuro y esotérico arte japonés hasta el fenómeno mundial que desde entonces y hasta ahora ha llegado a ser.
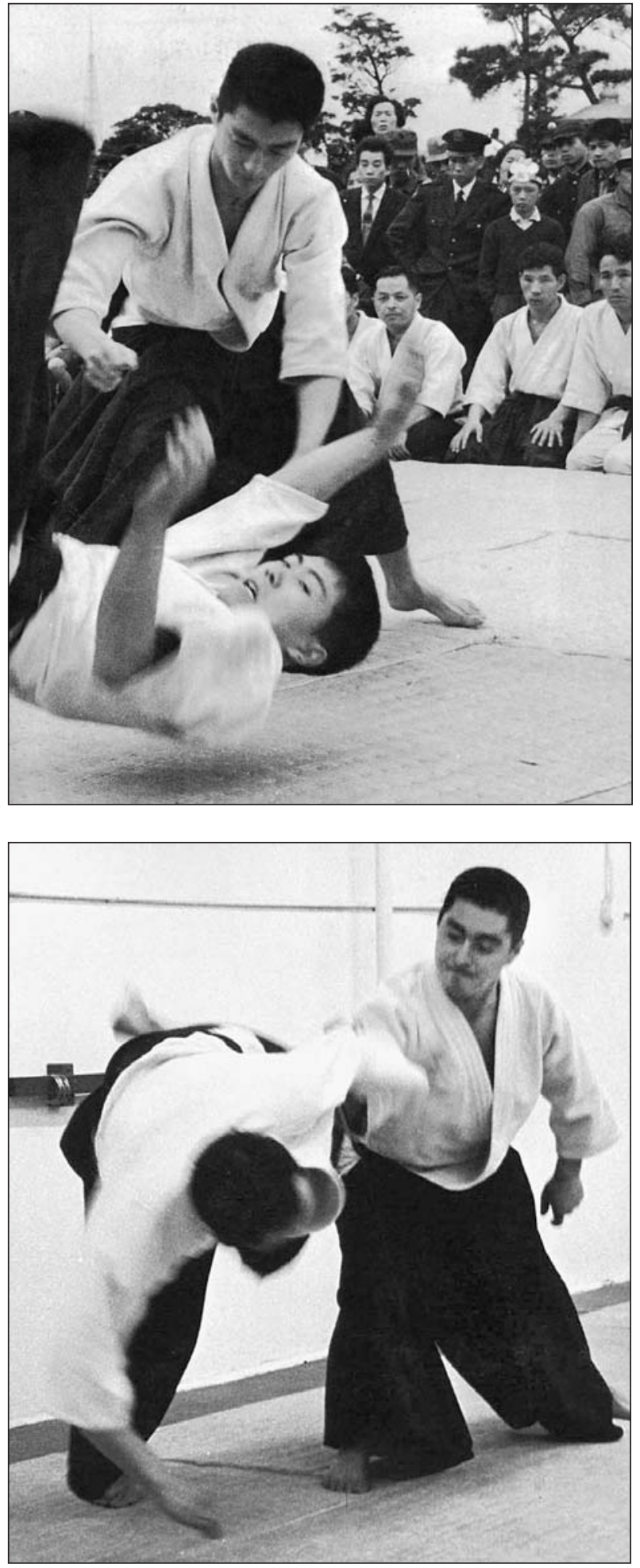

\section{El Aikido en el Japón de la Postguerra}

En los años inmediatamente posteriores a la Segunda Guerra Mundial, la práctica de las artes marciales fue prohibida en Japón por las fuerzas de ocupación comandadas por el general Douglas MacArthur. Cuando se levantó la prohibición, el aikido no era bien conocido ni siquiera por la mayoría de los japoneses. Fundado por Ueshiba Morihei, la sede del aikido fue una de las pocas construcciones que quedaron de pie en el área de Shinjuku, y fue usado como refugio para los vecinos que se quedaron sin hogar debido a la guerra. Si bien la vieja construcción de madera fue sustituida por una estructura más moderna, la sede y la residencia de Ueshiba todavía ocupan este lugar, uno de los puntos más elevados de Tokio.

Paulatinamente, mientras Japón empezaba a reconstruirse, una nueva generación de estudiantes fueron atraídos por las enseñanzas de Ueshiba. El grupo nuclear de estos estudiantes, los discípulos internos o aprendices del fundador, iban a tener una gran influencia en la difusión del aikido por todo el mundo. Este grupo incluía a Yamada Yoshimitsu, Kanai Mitsunari, Chiba Kazuo, y a Sugano Seiichi. Y entre ellos el principal responsable del desarrollo mundial del aikido es el maestro Yamada Yoshimitsu.

Yamada nació en Tokio el 17 de febrero de 1938, aunque durante la guerra pasó su primera infancia fuera de Japón. Vivió en la ciudad de Chiju, Korea, donde su padre era el gerente comercial de una compañía minera antes de su regreso a Japón. "Cuando regresé a casa [a Japón] fueron tiempos duros, pero ¿qué podía hacer? Fue una guerra. Lo miraba simplemente como algo positivo: fue bueno para mi”. Su padre llegó a ser profesor de economía comercial. Yamada describe a sus padres como "muy atípicos para ser japoneses en aquel tiempo, eran muy liberales". Su familia nunca le presionó para que entrara en los negocios, en el aikido, o en cualquier otra ocupación. "Ellos respetaban mi personalidad".

Se inició en el aikido a la edad aproximada de 13 años, cuando vio una demostración de Ueshiba. Hasta entonces él "no conocía nada sobre eso". El primo segundo de Yamada era Abe Tadashi, un antiguo discípulo de Ueshiba Morehei. "Mi padre y él eran primos. Sin embargo mis abuelos fallecieron cuando mi padre tenía siete años. Mi padre fue adoptado por la familia de Abe, de modo que yo le llamaba tío. Así que, 
aunque eran primos, se criaron como hermanos".

Yamada afirma que Abe Tadashi fue el primero en introducir el aikido en Occidente.

"El padre de Abe respetaba a Ueshiba Morihei y le apoyó económicamente. El Sr. Abe fue muy fiel a Ueshiba Morihei. Entiendo que Abe fuera el primero, incluso antes que Tohei [Koichi], en ir al extranjero para implantar el aikido, para lo cual fue hasta Francia. En ese momento era muy difícil ir al extranjero para los japoneses. Todavía estábamos bajo la ocupación americana y no era fácil viajar, pero él pudo ir usando los contactos de su padre con ciertas personas VIP. Pienso que tuvo una época dura porque nadie conocía qué era el aikido. En esos días, como todos los demás, él usaba judokas... como me pasó a mí. En los Estados Unidos tuve que utilizar a la gente de judo y kárate cuando comencé a hacer exhibiciones. No había nadie más" (Bernath \& Halprin, 1998).

Yamada nunca tuvo mucho interés por las artes marciales cuando era niño, "simplemente jugueteaba, no había nada serio", pero cuando se graduó en la escuela secundaria en 1955, sintió que "tenía que hacer algo para enderezar mi vida. Estaba viviendo la vida fácil y necesitaba algo de disciplina”. En esos días la práctica del aikido no estaba al alcance de cualquiera, y mucho menos el llegar a ser un discípulo interno.

"Simplemente necesitabas tener una buena presentación [referencias] para practicar. En el Japón de aquellos momentos el aikido no era en absoluto bien conocido. Sólo unas pocas personas sabían de su existencia. No lo anunciaban ni hacían ninguna demostración pública. Todavía recuerdo la primera demostración pública de aikido porque Ueshiba Morihei no les daba permiso para hacerla. Creo que [Ueshiba Kisshomaru, hijo del fundador] convenció a Ueshiba Morihei de que debía enseñarlo al público, pero él no quería... en los viejos tiempos, como todos los practicantes de las artes marciales, no quería enseñar sus técnicas a otros... cómo sacar la espada, lo que fuese... ellos no querían enseñar a cualquiera. Sólo debían enseñar a ciertos estudiantes porque no querían que sus enemigos lo viesen. Esto es por lo que antiguamente, si seleccionabas a gente para estudiar, debían tener primero una recomendación. Pero esto ha cambiado, del mismo modo que nuestro dojo se abrió al público. Pero no fue hasta entonces cuando nosotros comenzamos a realizar exhibiciones públicas... Pienso que él [Ueshiba Moriehi] no tenía elección, tenía que ganarse la vida" (Bernath \& Halprin, 1998).

\section{LA VidA COMO Discí́pulo de UeshibA MORIHEI}

Yamada cuenta que debido a su relación con Abe Tadashi, tuvo una "conexión especial" con Ueshiba Morihei y fue aceptado rápidamente.

"Era una situación única. Mi primer día de aikido fue mi primer día como uchi deshi. Otro en mi lugar habría entrado como cualquier otro estudiante y habría tenido que esperar para obtener permiso. En ese momento, aunque el aikido estaba empezando a abrirse más al público, sólo había unos pocos uchi deshi. Cuando comencé, Arakawa [Sadateru] y Tamura [Nobuyoshi] ya estaban allí, después yo mismo, y luego Chiba [Kazuo], Kurita [Yutaka], Kanai [Mitsunari] y Sugano [Seiichi]" (Bernath \& Halprin, 1998).

"Había muchos otros dentro y fuera, pero éstos son los únicos a los que considero el grupo legítimo. A veces algunos venían desde dojos filiales con una recomendación para la familia Ueshiba. Igual que sucede en mi propio dojo, que a veces la gente viene y está dos o tres meses".

Revista de Artes Marciales Asiáticas 9 Volumen 1 Número 1 (48-61) - 2006

\section{Nota del autor:}

el término japonés uchi deshi tiene más implicaciones que la de ser únicamente un estudiante interno. Aunque "aprendiz" puede ser un término sinónimo, pero un tanto basto, el autor prefiere "discípulo" debido a la íntima conexión sentida entre estos primeros estudiantes y el fundador. No tiene ningún tipo de implicación religiosa. 
Cuando le pregunté si tenía alguna historia sobre los otros uchi deshi, Yamada dijo "Seguro, toneladas de ellas", pero rechazó que se publicaran: "demasiado personales". Cuando pregunté si la personalidad de estos acólitos había cambiado con los años, se rió y dijo, "No, sigue siendo la misma, ellos nunca cambian". El entrenamiento como estudiante interno era muy riguroso, y los estudiantes mayores se comprometían a un "proceso de limpieza" que hacía todo incluso más difícil. Cuando le pregunté cómo era tratado por los mayores cuando empezó, Yamada respondió enfáticamente, “¡de mala manera!”. Más tarde corrige su afirmación diciendo "Ellos eran buenos. Pero el trato era áspero porque era muy diferente a mi estilo de vida habitual". [Juzgando por informes de otros que fueron uchi deshi por aquella época, el éxito de Yamada en la difusión del aikido refleja no solo su superior comprensión de la técnica sino también su considerable habilidad como diplomático].

"Ya Tada [Abe Tadashi] y Yamaguchi [Seigo] tenían una clase regular a la que enseñar, y creo que Tamura [Nobutoshi] también. En aquel tiempo Tohei Koichi era el Instructor Principal. Por esas fechas se fue a Hawai" (Bernath \& Halprin, 1998). "En cierto modo, era bueno porque era como una pequeña comunidad. Todos conocíamos de los demás. El dojo era una vieja construcción de madera. Tenía un buen ambiente. Ueshiba Morihei vivía allí. Él tenía una casa unida al dojo”. La rutina diaria de los uchi deshi era exigente. Había cinco clases cada día.

"Dormíamos en el tatami [colchonetas]. Las clases matinales empezaban a las 6.30, por cuanto teníamos que levantarnos temprano para abrir la puerta para la práctica, cocinar, y limpiar dentro del dojo y por fuera de la casa de la familia de Ueshiba. Claro que nosotros también cuidábamos de la familia de Ueshiba. La primera clase era impartida por el fallecido Kisshomaru Ueshiba, y a veces por el fundador, cuando estaba en Tokio".

Había clases adicionales a las 8.00 a.m., 3.00, 5.00, y a las 6.30 p.m., siendo la última la más numerosa "quizás 15 personas" en las colchonetas. "En el tiempo entre las clases regulares había clases privadas que las personas podían acordar con Ueshiba Morihei o con Tohei Koichi. Cada uno de nosotros debía estar disponible para hacer ukemis [caídas] durante estas clases" (Bernath \& Halprin, 1998).

El sistema de graduación de cinturones blanco/negro ya se usaba en esta época. [Hay cinco grados de cinturón blanco y, teóricamente, diez grados de cinturón negro: en el sistema original no se empleaban los cinturones de colores]. Yamada dice, "Nunca me sometí a ningún examen. Nunca preguntes por el grado. Simplemente espera a que el maestro te diga que estás preparado. Como los militares, no puedes condecorarte [promocionarte] a ti mismo... imenos aquí [en los Estados Unidos] donde hay personas que lo hacen!". Expresando sorpresa de que alguien pudiera sugerir un aumento de grado, Yamada opina que "la gente no debería decir cosas como, 'sólo para recordárselo, todavía soy tercer dan"'.

Yamada disfrutó de una relación muy próxima con el hijo de Ueshiba Morihei, Kisshomaru. Mientras algunos dicen que Kisshomaru no empezó a entrenar hasta bastante tarde en su vida, Yamada dice que esto no es cierto. "Él tuvo que parar durante algún tiempo debido a la Segunda Guerra Mundial, y más tarde tuvo que poner en marcha una empresa para mantener a su familia, pero el todavía venía y entrenaba en el dojo".

Después de aproximadamente cinco años viviendo en el dojo, Yamada se casó y se trasladó a un apartamento fuera de la sede. Se le consideraba todavía un uchi deshi, y se le asignaron responsabilidades de enseñanza en varias universidades y en otros clubes afiliados, incluyendo el enseñar a personal militar americano. Aunque no viajó muy frecuentemente con Ueshiba Morihei, obtuvo valiosas experiencias enseñando cuando acompañaba a Kisshomaru en las visitas a otros dojos a lo largo de Japón. 
Durante la guerra el fundador se trasladó a una pequeña granja en la prefectura de Iwama donde construyó una sala de entrenamiento y un santuario que fue [y aún es] sostenido por la familia Saito. Cuando le pregunté si alguna vez había viajado a Iwama con Ueshiba Morihei, contestó "Alguna vez, pero no me gustó. Estaban en el campo. Yo soy más un tipo de ciudad. Quise quedarme en Tokio". En un momento dado, Yamada atravesó una época de dudas, y de hecho dejó de practicar durante un corto periodo de tiempo. "Era joven y tenía algunas preguntas. Pensé que quizá el aikido fuese muy afeminado, ¿comprende? Pero estaba equivocado. Fue una buena educación para mi”. Yamada fue reacio a discutir los detalles de cómo le convencieron de la efectividad de este arte, diciendo con una sonrisa socarrona: "Descubrí por mi mismo que el aikido es un arte marcial poderoso".

\section{Viviendo con Ueshiba Morihei: "Debes 'Robar' la TÉCNICA”}

Ueshiba Morihei fue, obviamente, una persona muy religiosa. Un devoto seguidor de la fe Omotokyo. Sus creencias religiosas influyeron ciertamente en los fuertes cimientos éticos y morales por los que es conocido el aikido. Según la mayoría de relatos, nunca discutía sobre la técnica, y la mayoría de sus lecturas eran esotéricas hasta el punto de ser casi incomprensibles. Empezaba cada día con un la purificación ritual, cantando y rezando. Según Yamada, sin embargo, "aunque él era una persona muy religiosa, nunca nos obligó a hacer lo mismo. No creo que me hubiese quedado si lo hubiera hecho".

A lo largo de los años ha habido muchas historias que hablan de los supuestos poderes sobrenaturales de Ueshiba. Al preguntar sobre la habilidad de hacer milagros de Ueshiba Morihei, Yamada afirma, "No niego que fuese una persona especial, pero muchas historias han ido demasiado lejos. Esto no me gusta". Cuando le insistí para que contase un ejemplo de un supuesto milagro, Yamada respondió tajantemente: "No quiero que nadie se vuelva loco". Sin embargo, relata una vez que Ueshiba Morihei encontró algo que estaba perdido, y dijo que los dioses le habían indicado dónde encontrarlo. "Creo que esto fue bonito" (Yamada guarda para él sus creencias religiosas y siempre ha aceptado un acercamiento muy terrenal y técnico al arte. Raramente discute sobre el ki, permitiendo que esta energía se exprese por sí misma a través de sus técnicas).

Contrariamente al mito popular de que Ueshiba Morihei siempre irradiaba una santa conducta de paciencia y amabilidad, al parecer también "enseñaba a veces su temperamento". Según Kanai Mitsunari, Ueshiba Morihei “itenía una voz como el trueno!”. Cuando le preguntamos si Ueshiba Morihei se había enfadado alguna vez con él, Yamada dijo "claro, por cosas insignificantes. Yo no comprendía porqué se había enfadado. Sucedía y luego se le pasaba. Eso era lo bueno que tenía. Me gritaba y eso era todo. Se acababa".

En relación a las habilidades técnicas de Ueshiba Morihei, Yamada recuerda:

"Cuando le conocí estaba en la última etapa de su vida, y por eso su técnica era muy suave. A pesar de su edad tenía un montón de movimientos que me gustaban. Él solía decir 'todos los días mi técnica se transforma'. Es importante no abusar de este concepto de suavidad y pensar que el aikido tiene que ser obligatoriamente suave. Esto no es correcto. Si tenemos unos fundamentos sólidos y entrenamos muy duro, entonces, cuando nos hacemos mayores, nuestra técnica envejece, pero de ninguna manera se descuida. Esto se ha entendido muy mal. A veces la gente dice, por ejemplo, Ueshiba Morihei nunca te enseñaba a proyectar con la cadera, por lo tanto esto no debe ser parte del aikido. Pero es que simplemente están mirando la última parte de la vida de Ueshiba Morihei. Es simplemente una excusa. No importa lo que sea, pero seguimos haciendo budo. Podría ser más positivo, menos brutal, pero todavía es budo". 
YAMADA EN EL NEW YORK AIKIAI, CERCA DE 1973. Fotografía cortesía de Alicia Billman-Cordero. del aikido.

Según Yamada, Ueshiba Morihei nunca explicó "los aspectos técnicos"

"En la forma antigua de enseñanza el profesor no explicaba la técnica, debías copiarla del profesor. Esta es también mi filosofía. No obligo a ningún estudiante a hacer exactamente lo que yo hago, esto es imposible. Sencillamente demuestro el movimiento, y eres tú quien tiene que aprenderlo, asimilarlo. Desgraciadamente la mayoría de los estudiantes copian los malos hábitos del instructor, en cualquier caso. Es divertido, desearía que copiasen las cosas buenas".

\section{Los Primeros Tiempos en NueVa York}

Yamada vino a los Estados

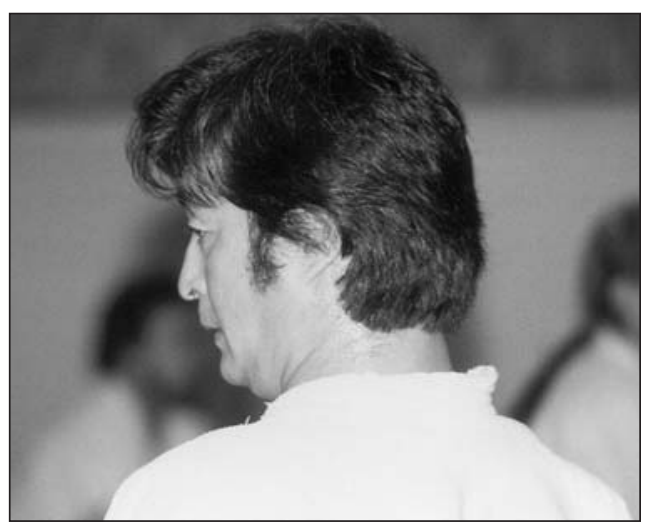

Unidos en 1964 con el propósito, en parte, de hacer una exhibición de aikido en el pabellón japonés de la Exposición Universal de Nueva York. "En un principio, Tohei Koichi iba a venir conmigo a la feria para hacer algunas demostraciones de aikido, pero tuvo un accidente y no pudo venir". Contrariamente a lo que cree la gente, Yamada no fue enviado por Ueshiba Morihei o por la sede. "Eso nunca sucedió", dijo,

"Fue una elección completamente personal. Yo siempre quise vivir en Nueva York. Sabía que Nueva York era mi tipo de ciudad. Y era exactamente igual que como había pensado que sería. Soy del tipo de personas que se adaptan muy fácilmente. Crecí con la cultura americana, ya sabes, música; pop y jazz, películas americanas... por eso no era nada nuevo para mi. No creo que hubiese permanecido tanto tiempo aquí si no amara la ciudad de Nueva York".

Dejando a su mujer e hijos en Japón durante los primeros años, Yamada vivió en el New York Aikikai, reviviendo un estilo de vida espartano. Puesto que había venido a los Estados Unidos con un visado cultural, fueron unos tiempos difíciles para Yamada debido a las autoridades de inmigración. Después de traerse a su familia a los EEUU se le obligó a que los devolviese a Japón durante varios años. Debido a las restricciones de su visado, no pudo volver a Japón para el funeral de Ueshiba Morihei en 1969 por miedo a que no le permitiesen volver a los EEUU. Yamada expresa su enorme pesar debido a tener que abandonar a su familia. "Me siento mal con mi familia. No tengo muchos recuerdos con los niños. Estuvimos separados y después de que volvieran estaba muy ocupado y no éramos tan afortunados como somos ahora. Simplemente no podía llevarlos a todas las partes a las que yo iba. Éste es mi gran pesar, el tiempo que no disfruté de mi familia” (Bernath \& Halprin, 1998).

"Al principio, todos los estudiantes eran exjudokas y exkaratekas. Eran los únicos que estaban interesados". Muchos, si no la mayoría de los practicantes de artes marciales en los EEUU durante la década de los 50 y principios de la década de los 60, eran antiguo personal militar que habían conocido estas artes durante las giras de la posguerra en Oriente. Incluimos al mencionado Rodney Grantham entre esos pioneros para el caso del aikido, quien había empezado a practicar judo en 1950, consiguiendo su cinturón negro mientras estuvo destinado en Japón durante la guerra de Corea. 
Grantham fundó la "Black Belt School of Judo" ["Escuela de Judo Cinturón Negro"] en Atlanta durante la década de los 60. La primera vez que tuvo contacto con el aikido fue cuando le ofrecieron la oportunidad de ser proyectado por el legendario Tohei Koichi. Pronto abandonó completamente el judo para estudiar aikido bajo la dirección de Yamada. En 1967 fundó el Aikido Center of Atlanta [Centro de Aikido de Atlanta], que aún sigue funcionando hoy en día.

"No nos anunciábamos públicamente. Había también gente del taijiquan. En aquellos tiempos había empezado el boom del kárate. Tenía buenas relaciones con todos los profesores de kárate americanos, así que cada vez que ellos tenían un torneo, siempre me invitaban a ir y hacer una exhibición en el Madison Square Garden y en otros lugares. Casi todas las semanas me invitaban. Por supuesto que no me pagaban, pero era una buena oportunidad para dar a conocer el aikido... Por eso ya no me gusta hacer demostraciones. Hice tantas que estoy cansado de ellas, fue demasiado. Pero entonces nosotros aprovechábamos cualquier oportunidad. Una vez hice una demostración en las calles del sur del Bronx, en el hormigón. Hacía un tiempo invernal y por eso yo llevaba guantes negros. Recuerdo a un tipo que estaba diciendo cosas como, ‘¡Oh! ¡Es un asesino, tiene guantes negros!' (riéndose). Por entonces gran cantidad de gente tenía ideas descabelladas sobre las artes marciales. Ellos copiaban lo que hacía Bruce Lee en la serie de televisión en que participaba (Green Hornet [El Avispón Verde]). Esta serie ayudó mucho a que las artes marciales ganasen popularidad, más interés. Hice una demostración junto a otro tipo, una estrella del cine... ahora hace de Ranger de Texas... si, eso es: Chuck Norris. Un buen tipo. Hicimos una demostración una vez en el Hilton de New york. Le gustaba el aikido" (Bernath, 1998).

En ese momento había sólo un puñado de estudiantes que practicaban el aikido en Nueva York, incluyendo al actual presidente de la USAF [Fuerza Aeronáutica de los Estados Unidos] Mike Abrams. Abrams, quien ahora ostenta el grado de $7^{\circ}$ dan, recuerda, "Solíamos preguntar a Yamada todo tipo de preguntas inteligentes como: ¿Qué haría si es atacado por seis tipos a caballo con ametralladoras?... isabes?, cosas tan inteligentes como estas" (Abrams, 1994).

\section{El Desarrollo del Aikido}

Desde el momento en que llegó a los EEUU, Yamada viajó mucho, enseñando en cursillos y ayudando en los comienzos de nuevos dojos. "Incluso entonces viajé mucho, iba a Boston todos los lunes antes de que viniera Kanai [Mitsunari]". (Kanai llegó a los EEUU varios años después de Yamada. Llegó a ser el Instructor Principal de la segunda escuela de aikido más antigua de la costa este, The New England Aikiai. Kanai falleció en 2004). Según comenzaron a proliferar los dojos a lo largo de la costa este, Yamada empezó a viajar más y más. "No en avión, como ahora. En aquellos días el autobús era mi transporte". Yamada podía viajar en autobús desde Nueva York a Atlanta para enseñar en el dojo de Rodney Grantham. Yamada seguía entonces hacia Titusville, Florida, para enseñar en la escuela de aikido de otro pionero, el Doctor Tom Walker. Un antiguo estudiante del New York Aikikai, Alice Billman Cordero (ahora 5o dan) recuerda: "Nos amontonábamos todos en el viejo coche de alguien y conducíamos hasta Conética en medio de una tormenta de nieve. Todo era muy pequeño en aquellos días. Todos nos conocíamos. Yo sólo era un adolescente y [Yamada] me acompañaba en el metro para asegurarse de que llegaba sano y salvo a casa" (correspondencia personal, 2003). 


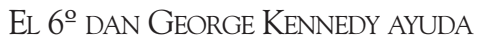
A DIFUNDIR EL ESTILO DE AIKIDO DE YAMADA EN ATLANTA, GEORGIA.

KENNEDY BLOQUEA Y EVITA UN PUÑETAZO, GOLPEANDO SIMULTÁNEAMENTE LA CABEZA DEL ATACANTE. RÁPIDAMENTE PROGRESA HACIA UNA LUXACIÓN

DE MUÑECA Y CODO, LLEVANDO AL SUELO AL ATACANTE.

Fotografías cortesía de Jeremy Wojcik.

UN ESPECIAL AGRADECIMIENTO A

Barry Kellerman, Aaron MacNeil, Ted Banta, Patrick HARDESTY y SKIP CHAPMAN POR SU AYUDA EN LA PARTE TÉCNICA.
A lo largo de los años, la influencia de Yamada ha dejado una huella indeleble en el arte a nivel mundial. Su programa de seminarios le lleva a los lugares más alejados del globo, impartiendo incluso dos seminarios distintos en el mismo fin de semana. En 1996 se constituyó la Federación Latino Americana de Aikido con el visto bueno de Yamada, institución que suma millares de miembros de diferentes organizaciones. Con todo, ha mantenido un gran nivel de excelencia técnica mientras guiaba el desarrollo del aikido por todo el mundo. Hablando sobre su rol en el desarrollo del aikido, Yamada cuenta: "No sabía qué pasaría. Fue bonito tener un pequeño grupo, pero sabía que no duraría para siempre. Ahora soy feliz de ver a tantas personas involucradas. Es un buen arte. Es beneficioso para la vida de los practicantes, por lo tanto, creo, que es un buen logro".

Cuando le preguntamos sobre la calidad del aikido de ahora comparándolo con la del aikido de los viejos tiempos, contesta,

"El aikido es muy individual, muy personal, depende de cómo lo interprete cada instructor, por eso es muy difícil mantener un nivel alto... pero lo intento. El aikido es diferente en cada lugar del mundo debido a la mentalidad peculiar de cada país. Es muy difícil intentar mantener a todos juntos. Lo intento, pero no sé si estoy haciendo un buen trabajo o no. Siempre que juntas a tanta gente tienes problemas, nace el conflicto. Como líder he de tener una mente muy flexible. Básicamente, respeto a cada individuo; no me importa quien sea. Mi filosofía es que debes tener confianza, respeto, y ser justo, estas son las cosas más importantes tanto para el profesor como para el estudiante".
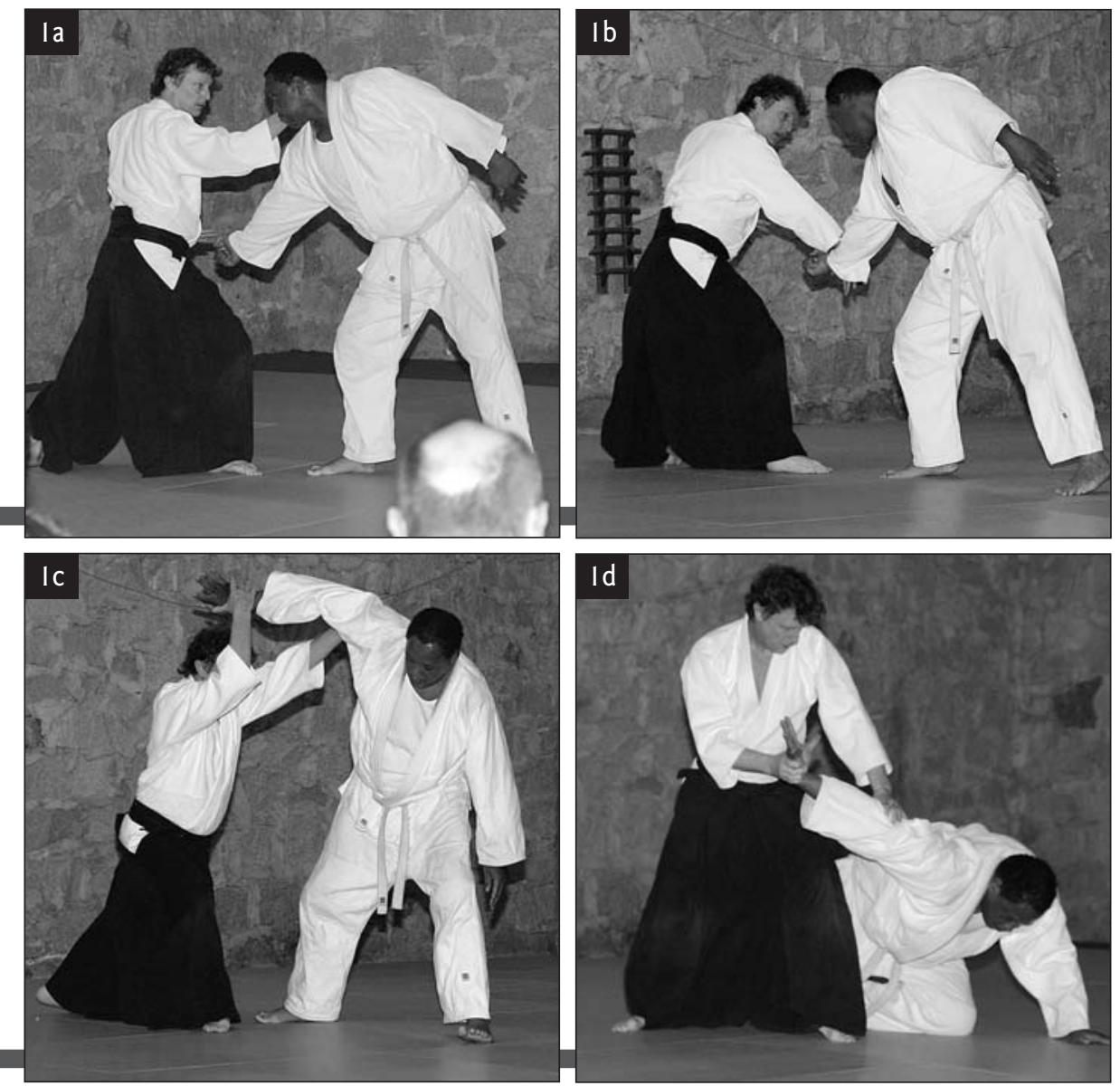

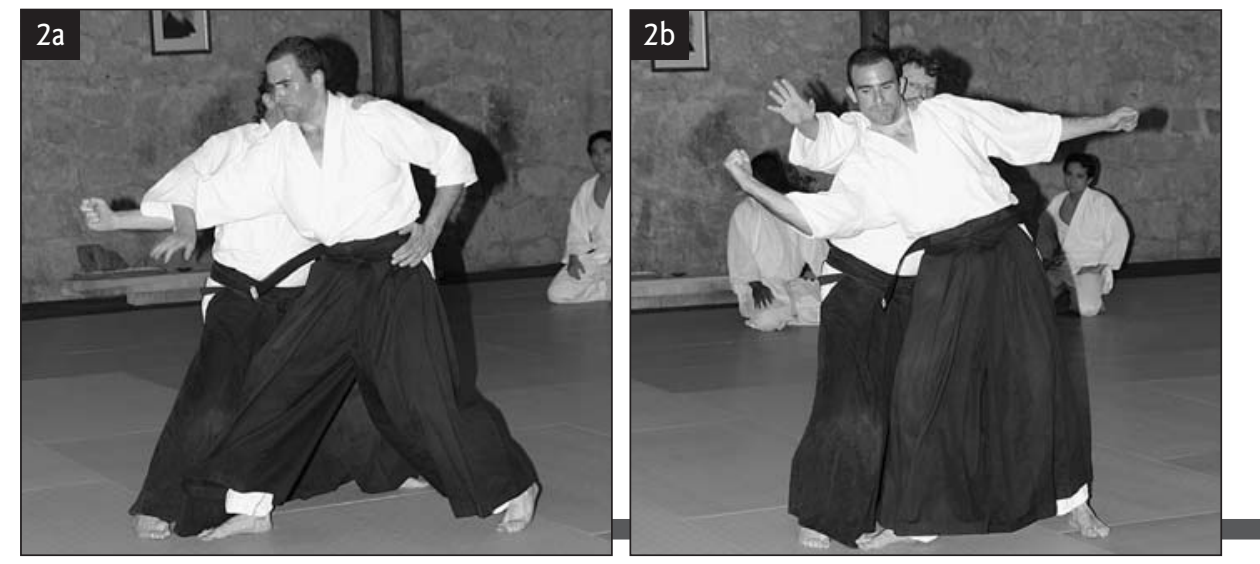

GEORGE KenNEDY ESQUiva HACIA FUERA UN INTENTO DE GOLPEO, DESEQUILIBRANDO INMEDIATAMENTE A SU ATACANTE PARA PROYECTARLO DE ESPALDAS SOBRE EL SUELO.

Fotografías cortesía de Jeremy Wojcik.
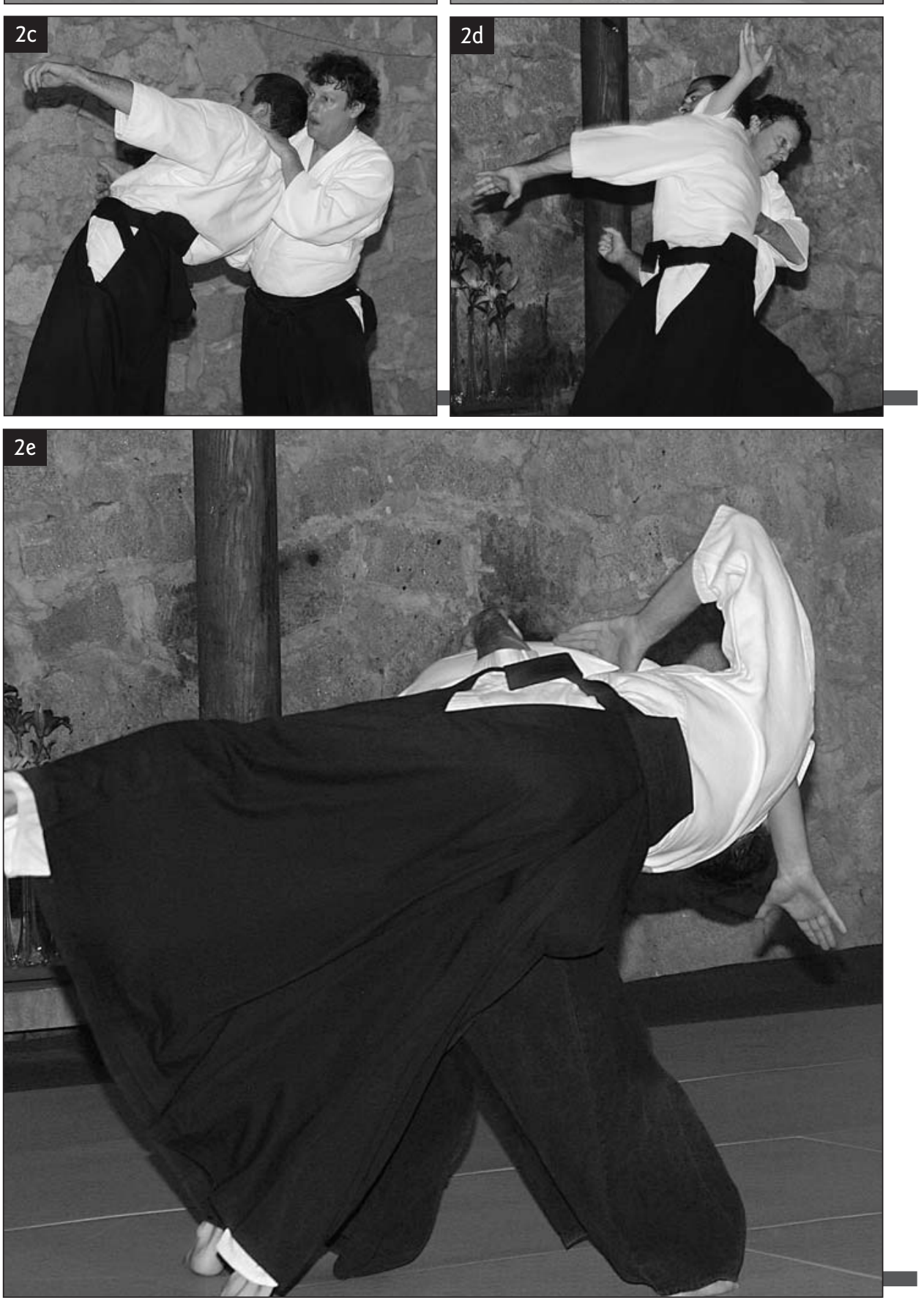
UNA DEMOSTRACIÓN DE YAMADA EN EL AiKIDO CENTER OF ATLANTA, MARZO DE 2005. Fotografías cortesía de Jeremy Wojcik.
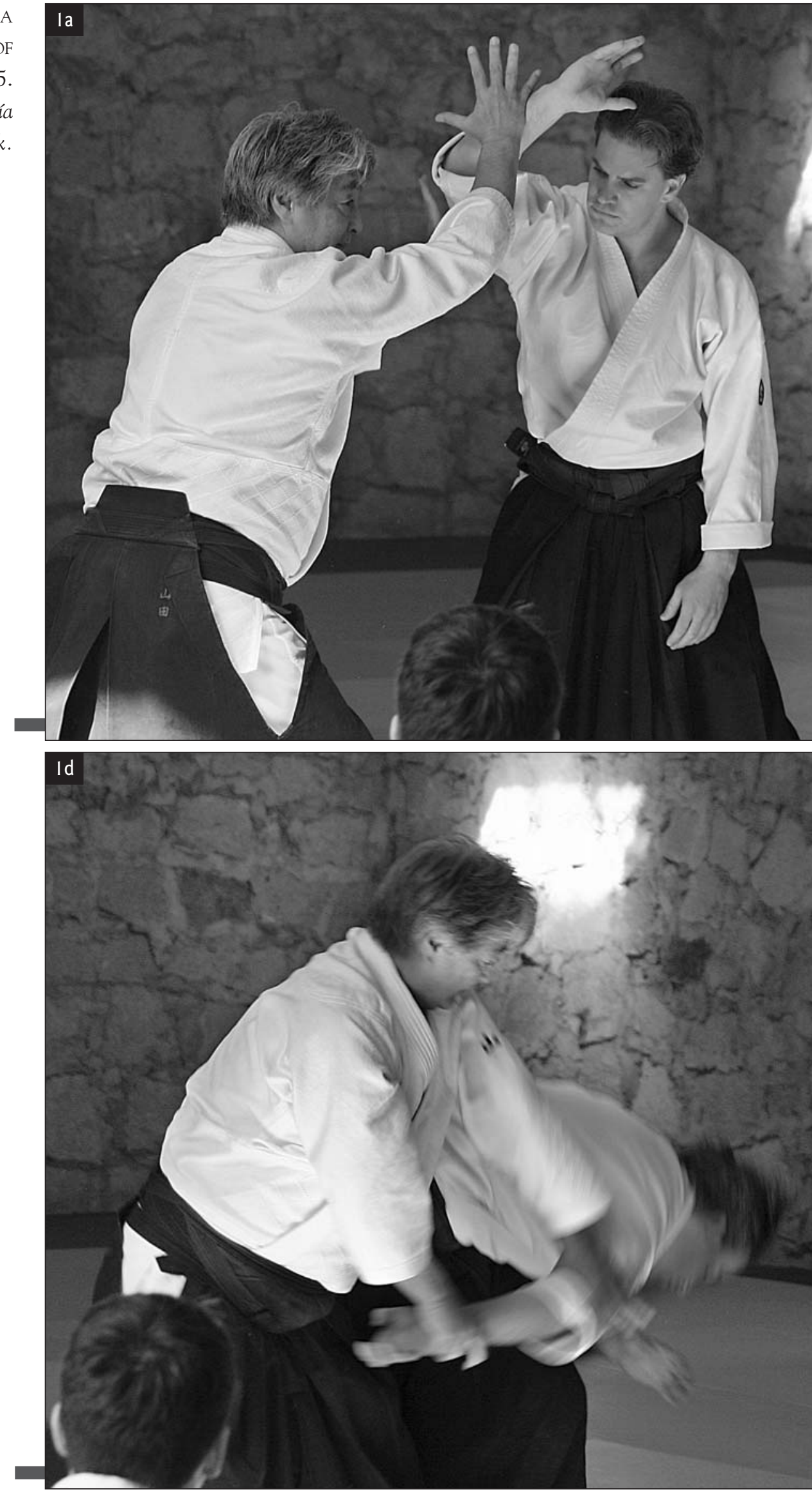

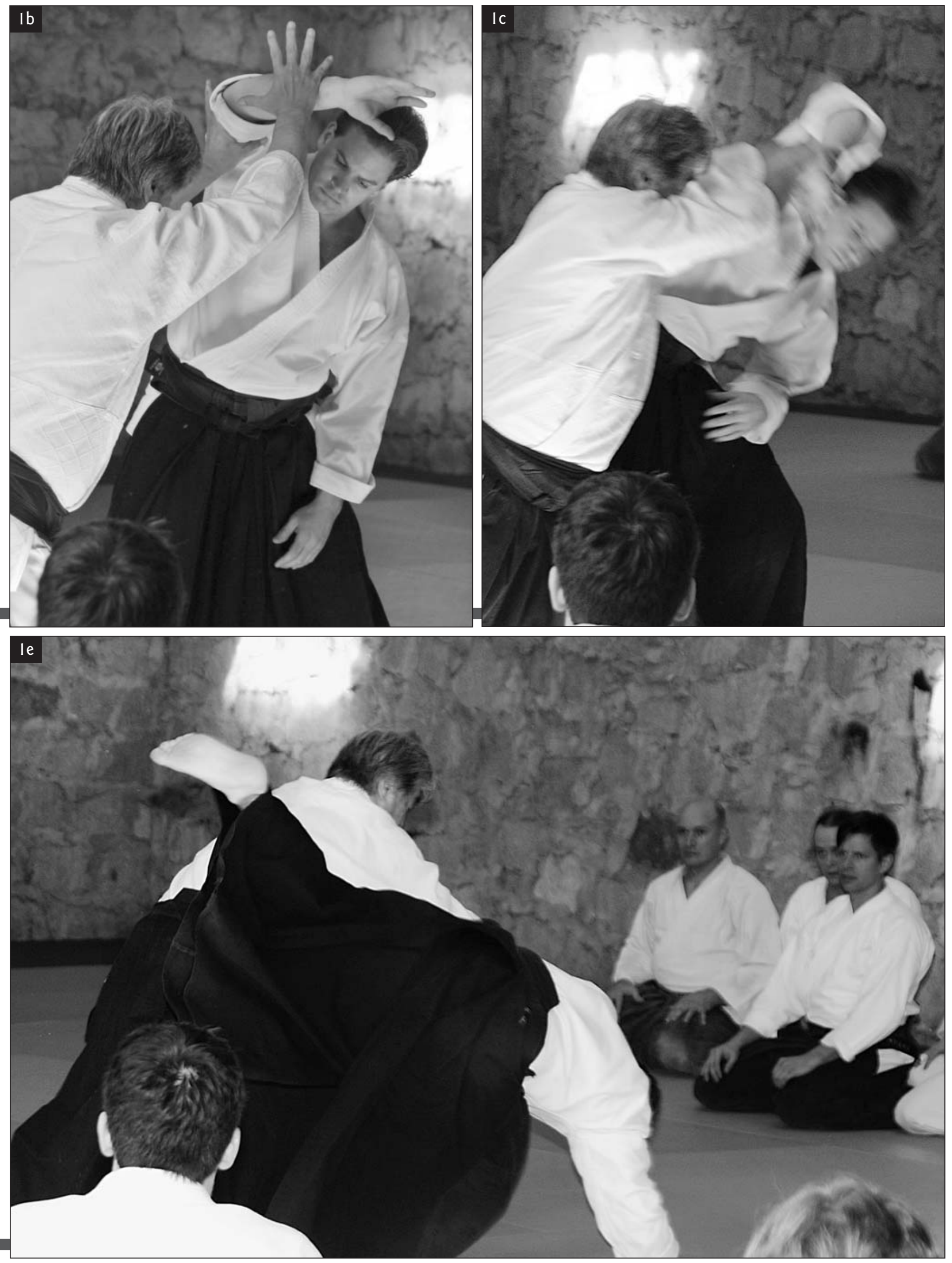

Revista de Artes Marciales Asiáticas 9 Volumen 1 Número 1 (48-61) - 2006 


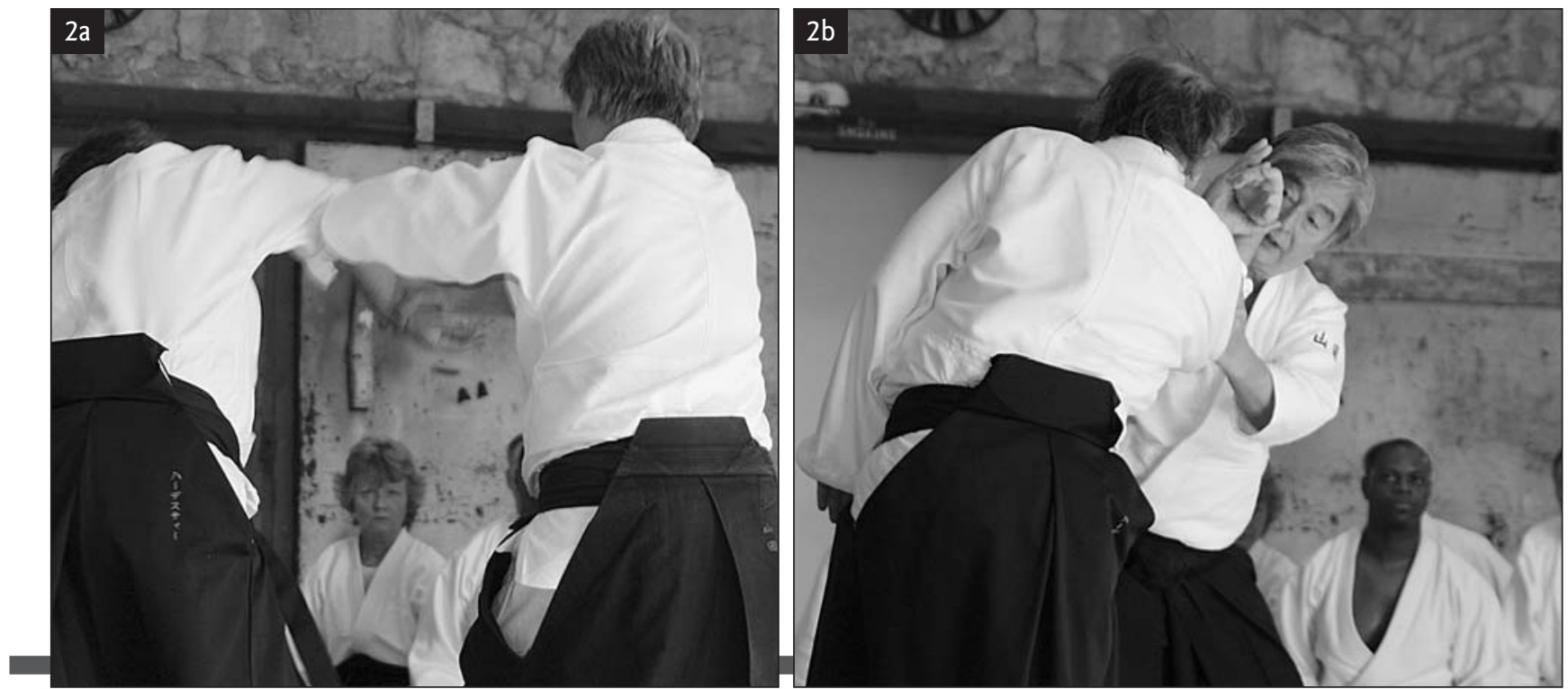

UNA DEMOSTRACIÓN DE YAMADA EN EL AIKIDO CENTER OF ATLANTA, MARZO DE 2005. Fotografías cortesía de Jeremy Wojcik.
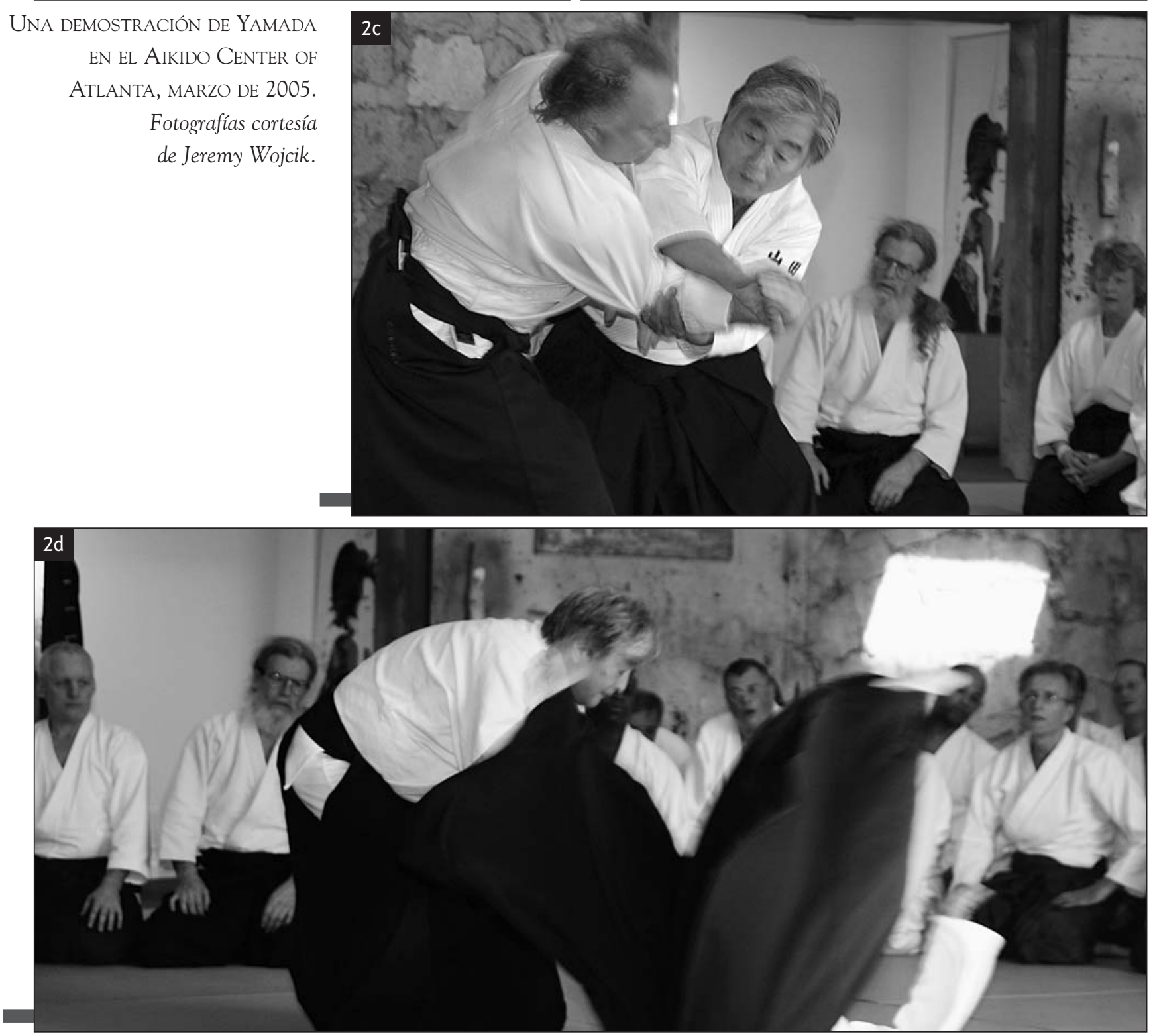

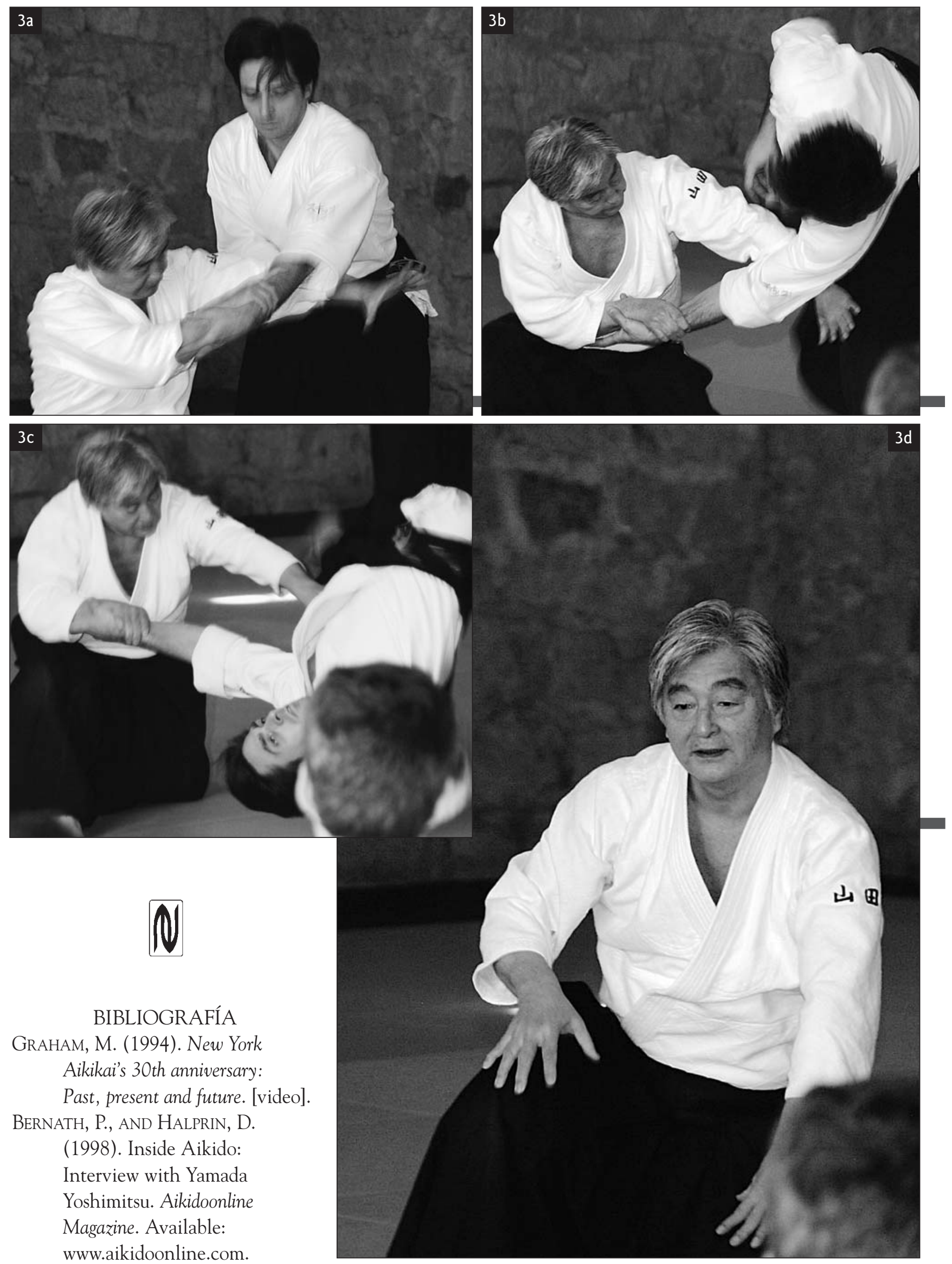

Revista de Artes Marciales Asiáticas 9 Volumen 1 Número 1 (48-61) - 2006 